Gynäkologe 2006 · 39:820-823

DOI 10.1007/s00129-006-1899-x

Online publiziert: 12 . Oktober 2006

(c) Springer Medizin Verlag 2006

\section{Redaktion}

R. Kreienberg, Ulm

\author{
H. Köhler ${ }^{1} \cdot$ C. Adersen ${ }^{2} \cdot$ M. Glenck ${ }^{3}$ - B. von Castelberg ${ }^{1}$ \\ ${ }^{1}$ Frauenklinik Maternite, Stadtspital Triemli, Zürich \\ ${ }^{2}$ Institut für Pathologie, Stadtspital Triemli, Zürich \\ ${ }^{3}$ Institut für Radiologie, Stadtspital Triemli, Zürich
}

\title{
Zunehmende Asymmetrie der Brust ohne klaren Palpationsbefund
}

\section{Anamnese}

Bei uns wurde eine 34-jährige Primipara vorstellig mit der Frage nach der Ursache einer Brustasymmetrie (links $>$ rechts). Sie berichtete von einer graduell zunehmenden Vergrößerung der linken Brust seit der Geburt ihres einzigen Sohns vor 3 Jahren. Schmerzen oder eine auffällige Mamillensekretion wurden verneint. Die Familienanamnese für benigne und maligne Brusterkrankungen sei negativ. Bisher keine Diagnostik oder Therapie.

\section{Klinischer Befund}

\section{Inspektion, Palpation}

Inspektorisch imponierte die linke Mamma vergrößert. Retraktionsphänomene waren nicht nachweisbar, die Mamille präsentierte sich unauffällig. Bei der Palpation konnte ein Tumor vom umgebenden Brustdrüsenkörper nicht sicher abgegrenzt werden. Die regionären Lymphknotenstationen waren unauffällig. Die rechte Mamma inklusive der Lymphabflussgebiete zeigte keinen auffälligen Befund (• Abb. 1).

\section{Diagnostik}

\section{Mammographie}

$10 x 15 \mathrm{~cm}$ messende, homogene, scharf begrenzte Transparenzminderung im oberen äußeren Quadranten links (BI-RADSKategorie 4) (• Abb. 2a, b).
Differenzialdiagnosen aus radiologischer Sicht:

- Phylloidestumor

- Riesenfibroadenom

- Muzinöses Mammakarzinom

\section{Mammasonographie}

Wegen der Ausdehnung des Befundes musste die 5-MHz-Abdominalsonde verwendet werden.

Es zeigte sich eine glatt begrenzte, hypoechogene, weitgehend homogene Raumforderung mit horizontaler Ausrichtung (Dimension 4,5X7,3x8,8 cm) und Verdrängung der umgebenden Strukturen. Im Zentrum des Tumors bestand eine verstärkte Perfusion. Mithilfe der Sonopalpation konnte eine gute Verschiebbarkeit zu den Nachbarstrukturen demonstriert werden. Pathologische axilläre Lymphknoten links waren nicht darstell- bar. BI-RADS-Klassifikation (analog) 3

(• Abb. 3a, b).

\section{Stanzbiopsie links}

Laut Pathologie nicht konklusiv, kein sicherer Nachweis eines Tumors.

\section{Therapie und Verlauf}

Aufgrund der Größe des Tumors und seiner unklaren Dignität entschlossen wir uns im Einverständnis mit der Patientin zur Exzision des Befundes. Es wurde eine Tumorektomie mit sparsamer Resektion überschüssiger Haut durchgeführt (- Abb. 4, 5).

Der postoperative Verlauf gestaltete sich problemlos, die Patientin konnte am 2. Tag nach dem Eingriff mit reizlosen Wundverhältnissen entlassen werden (• Abb. 6).

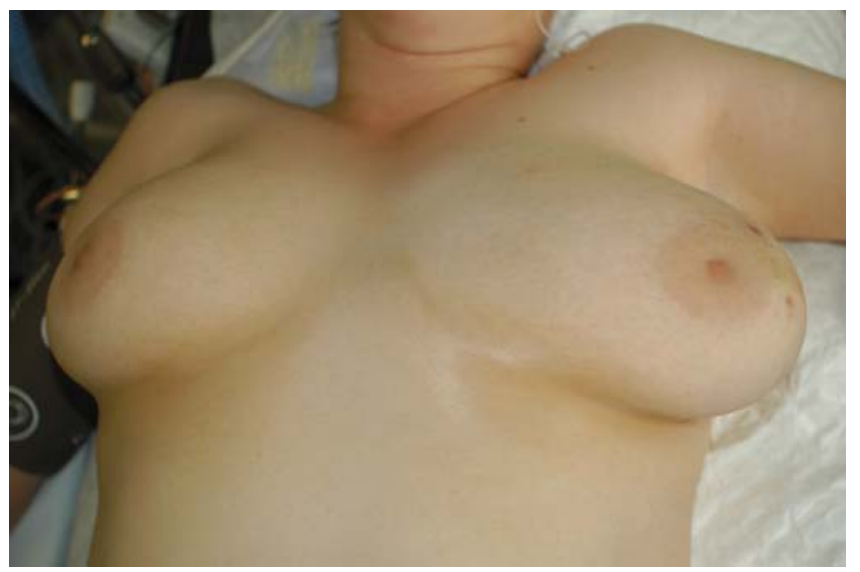

Abb. $1<$ Präoperativer Situs, Einstichstelle nach Stanzbiopsie erkennbar 


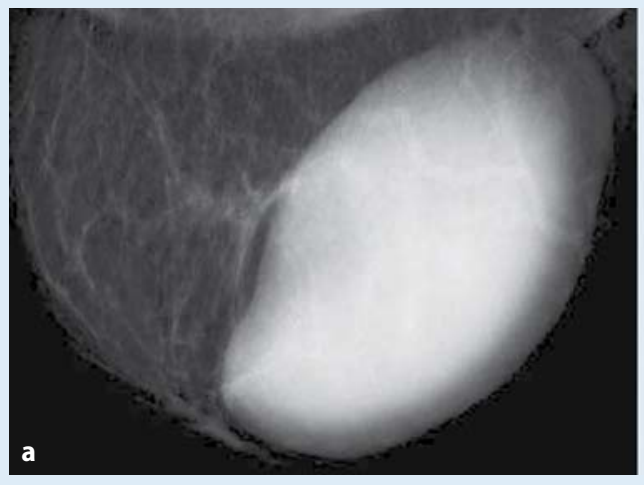

Abb. 2a, b $\Delta$ Mammographie links (CC- und MLO-Projektion)
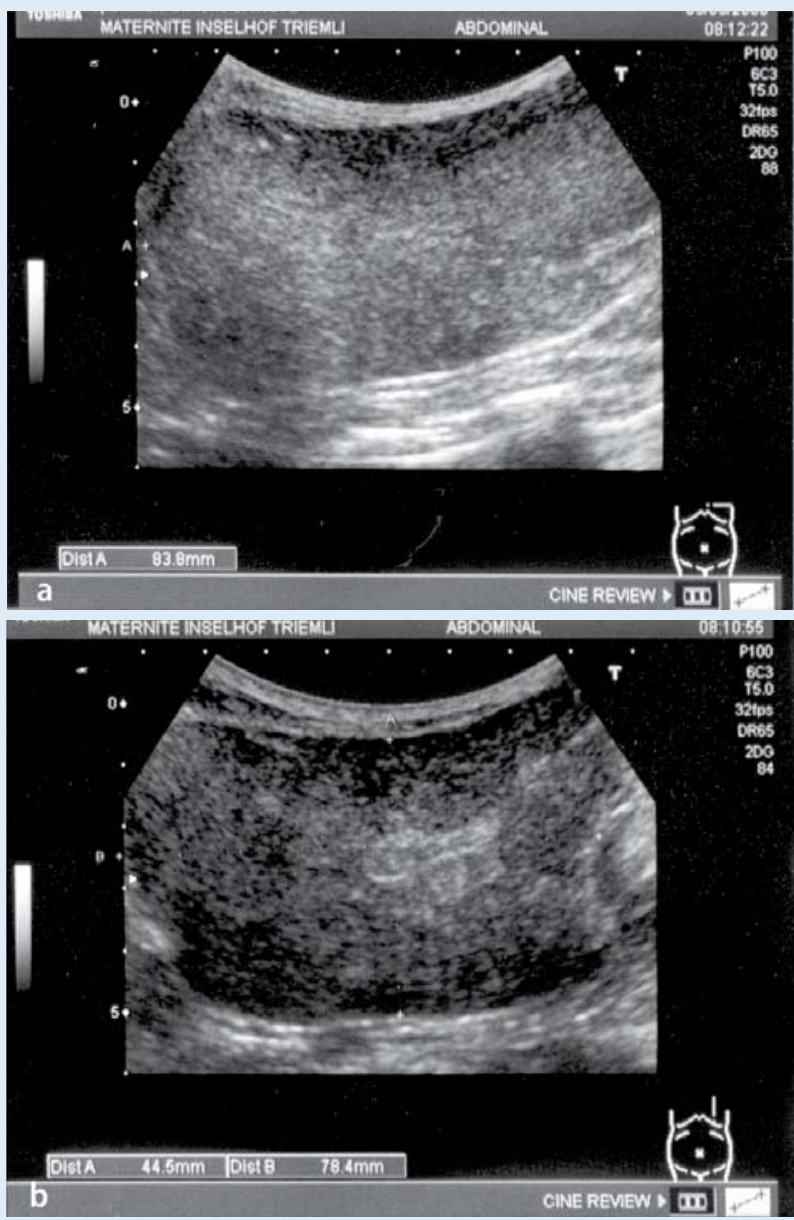

Abb. 3a, b $\Delta$ Mammasonograhie links )

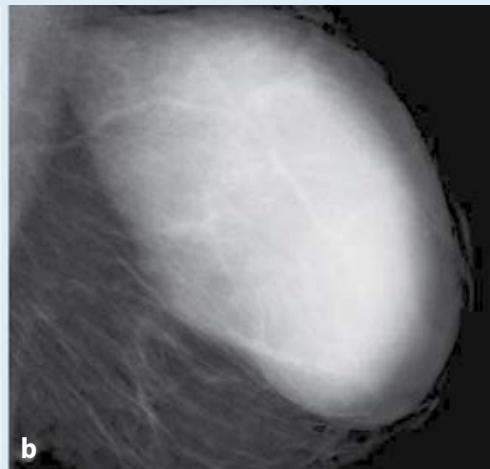




\section{> Diagnose: Subtotal sklerosiertes Fibroadenom (Giant Fibroadenoma) der linken Mamma ohne Anhaltspunkte für Malignität}

Gewicht: 332 g, Dimension 11,5x9x5,5 cm

\section{Diskussion}

Die 34-jährige Patientin präsentierte sich mit einer zunehmenden indolenten Vergrößerung der linken Brust. Bemerkenswert war die Diskrepanz zwischen der Ausdehnung des Tumors und dem nahezu unauffälligen Palpationsbefund. In den bildgebenden Untersuchungsverfahren konnte jedoch klar ein solider Tumor diagnostiziert werden. Die mammasonographischen und mammographischen Befunde deuteten auf ein benignes Wachstumsmuster hin, obgleich ein Mammakarzinom nicht sicher auszuschließen war. Die stanzbioptische Untersuchung war nicht konklusiv. Differenzialdiagnostisch kamen folgende Läsionen in Frage [3, 4]:

- Cystosarcoma phylloides

- Giant Fibroadenoma

- Lipom

- Hamartom

- Tubuläres Adenom

- Mammakarzinom

- Andere seltene Tumoren

Die definitive histopathologische Diagnose lautete: subtotal sklerosiertes Fibroadenom (Giant Fibroadenoma).

Fibroadenome gehören zu den häufigsten benignen soliden Brusttumoren der jungen Frau [1, 4], in der hier berichteten Größe treten sie allerdings wesentlich seltener auf. Im Gegensatz zum Cystosarcoma phylloides sind Riesenfibroadenome häufig bereits bei noch adoleszenten, dunkelhäutigen Frauen zu finden [2]. Typischerweise präsentieren sich die Frauen mit einer unilateralen, schmerzlosen Brustvergrößerung. Die Patientinnen berichten meist über ein rasches Wachstum. Palpatorisch ist der Tumor oft schwer abgrenzbar, eine Dilatation oberflächlicher kutaner Venen über dem Tumor ist häufig präsent. Eine chirurgische Exzision ist sowohl wegen der Tumorgröße als auch zur Sicherung der Dignität unerlässlich.

\section{Fazit für die Praxis}

- Das Riesenfibroadenom (Giant Fibroadenoma) ist eine Erkrankung der jungen Frau.

- Eine neu aufgetretene Größenasymmetrie der Brust berechtigt zur Durchführung einer bildgebenden Mammadiagnostik, auch wenn der Palpationsbefund blande erscheint.

- Die Stanzbiopsie ist bei Riesenfibroadenomen häufig nicht konklusiv.

- Die Therapie der Wahl ist die chirurgische Exzision. Eine Reduktion der überschüssigen Haut sollte vorsichtig angewendet werden, da sich das druckatrophierte umliegende normale Drüsengewebe innerhalb von 6 Monaten spontan erholt.

\section{Korrespondierender Autor}

Dr. H. Köhler

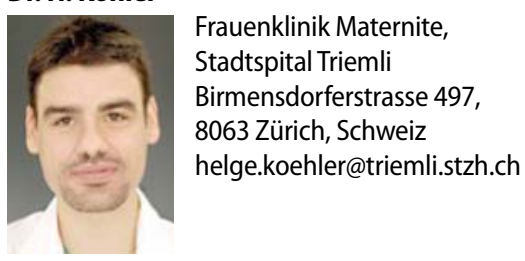

Interessenkonflikt. Es besteht kein Interessenkonflikt. Der korrespondierende Autor versichert, dass keine Verbindungen mit einer Firma, deren Produkt in dem Artikel genannt ist, oder einer Firma, die ein Konkurrenzprodukt vertreibt, bestehen. Die Präsentation des Themas ist unabhängig und die Darstellung der Inhalte produktneutral.

\section{Literatur}

1. Musio F, Mozingo D, Otchy DP (1991) Multiple giant fibroadenoma. Am Surg 57: 438-441

2. Magnoni P, Nardi F (1996) Giant fibroadenoma of the breast. Its clinical picture and differential diagnosis. A report of a clinical case. Minerva Chir 51: 71-75

3. Raganoonan C, Fairbain JK, Williams S, Hughes LE (1987) Giant breast tumours in adolescence. Aust NZ J Surg 57: 243-247

4. Santen RJ, Mansel R (2005) Benign breast disorders. N Engl J Med 353: 275
Leser fragen - Experten antworten

Haben Sie Fragen aus Ihrer täglichen Praxis? Dann wenden Sie sich an die Herausgeber der Rubrik "Leser fragen - Experten antworten". Ausgewiesene Experten des jeweiligen Fachgebiets beantworten Ihre Fragen kompetent und schnell. Beiträge von allgemeinem Interesse werden anschließend ohne Nennung Ihres Namens in „Der Gynäkologe" publiziert.

Bitte schicken Sie Ihre Fragen am einfachsten per E-Mail mit dem Betreff

„Der Gynäkologe - Leserfrage“

an die Rubrikherausgeber:

- Prof. Dr. Klaus Diedrich

- PD Dr. Michael Friedrich

Klinik für Frauenheilkunde und Geburtshilfe Universitätsklinikum Schleswig-Holstein

Campus Lübeck

Ratzeburger Allee 160

23538 Lübeck

E-Mail: diedrich@medinf.mu-luebeck.de 
Hier steht eine Anzeige.

算 Springer 